\title{
Emerging problems regarding severity assessment and treatment strategies for patients with pneumonia: controversies surrounding the HCAP concept
}

\author{
Yuichiro Shindo $\cdot$ Yoshinori Hasegawa
}

Received: 12 April 2011 / Accepted: 28 April 2011/Published online: 18 May 2011

(C) SIMI 2011

$\begin{array}{ll}\text { Abbreviations } \\ \text { ATS } & \text { American Thoracic Society } \\ \text { CAP } & \text { Community-acquired pneumonia } \\ \text { HCAP } & \text { Health care-associated pneumonia } \\ \text { ICU } & \text { Intensive care unit } \\ \text { IDSA } & \text { Infectious Diseases Society of America } \\ \text { MDR } & \text { Multidrug-resistant } \\ \text { MRSA } & \text { Methicillin-resistant Staphylococcus aureus } \\ \text { PDR } & \text { Potential drug-resistant } \\ \text { PSI } & \text { Pneumonia Severity Index } \\ \text { SCAP } & \text { Severe community-acquired pneumonia }\end{array}$

In this issue of Internal and Emergency Medicine, Falcone et al. [1] validate the widely used predictive scoring systems for assessing the severity of pneumonia. These include the Pneumonia Severity Index (PSI) [2], CURB-65 (confusion, uremia, respiratory rate, low blood pressure, 65 years of age or older) [3], and severe communityacquired pneumonia (SCAP) scores [4] for hospitalized patients with community-acquired pneumonia (CAP) and health care-associated pneumonia (HCAP) who were enrolled in a prospective multicenter cohort study in Italy [5]. The authors find that PSI, CURB-65, and SCAP scores have a good performance for patients with CAP, but are

Y. Shindo and Y. Hasegawa contributed equally to this work.

Y. Shindo

The Institute for Advanced Research, Nagoya University,

Nagoya, Japan

Y. Shindo $(\bowtie) \cdot$ Y. Hasegawa

Department of Respiratory Medicine, Nagoya University

Graduate School of Medicine, 65 Tsurumai-cho, Showa-ku,

Nagoya 466-8550, Japan

e-mail: yshindo@med.nagoya-u.ac.jp less useful for patients with HCAP, particularly those categorized as low risk. Furthermore, they describe that inclusion in the severe classes of PSI, CURB-65, and SCAP scores and receiving an empirical treatment that does not adhere to international guidelines, as documented in the 2005 or 2007 American Thoracic Society (ATS)/ Infectious Diseases Society of America (IDSA) [6, 7], prove to be risk factors independently associated with inhospital mortality or development of adverse events, including death, septic shock, and the need for a transfer to an intensive care unit (ICU).

The PSI and CURB-65 scores were originally used as predictive scoring systems to identify patients who could receive outpatient treatment, as low scores were associated with low mortality [2, 3]. However, the reported sensitivity and specificity of high scores for severe CAP, related to high mortality or adverse events, show that these systems are not very useful [7]. Therefore, an alternative scoring system, SCAP scores, was designed to identify patients with severe CAP [4]. The enrolled patients with CAP and HCAP in this Italian study required hospitalization [5]. Therefore, it was suitable to assess patients with severe pneumonia who were likely to have a fatal course, and not who could receive outpatient treatment.

When the PSI, CURB-65, and SCAP scores were developed, the concept of HCAP was not widely used. Thus, a substantial number of HCAP patients were previously defined as having CAP. A Japanese retrospective study shows that areas under the receiver operating characteristic curves for the 30-day mortality in predictive rules, including those for CURB-65, are greater in patients with newly defined CAP [7], in which HCAP is excluded, than in those with previously defined CAP [8]. This study shows similar results; high proportions of mortality and adverse events among HCAP patients with low severity 
scores determined by PSI, CURB-65, and SCAP suggesting that the predictive rules for CAP are not useful for patients with HCAP. Over the past several years, the reasons for why HCAP should be distinguished from CAP have been based on evidence that the proportion of occurrence of potentially drug-resistant (PDR) pathogens [e.g., Pseudomonas aeruginosa, extended-spectrum $\beta$-lactamase-producing Gram-negative bacteria, and methicillinresistant Staphylococcus aureus (MRSA)] and the receipt of inappropriate empirical treatments in HCAP patients are higher than those in CAP patients [9, 10]. Therefore, the results of this study provide a different perspective to support the necessity for using the HCAP concept as a distinct entity.

Why are PSI, CURB-65, and SCAP scores not useful for patients with HCAP? The reasons may depend on the following: (1) there may be confounding factors related to mortality or adverse events that are not included in their predictive rules (e.g., parameters for activities of daily living) and (2) cut-off values for continuous variables that are included in these rules are different for patients with CAP and HCAP. Moreover, it is undeniable that treatment restrictions for patients with HCAP exist, as pointed out by Ewig et al. [11]. Therefore, additional studies designed to analyze risk factors for mortality or adverse events in patients with HCAP are required considering the above factors.

Falcone et al. describe that the receipt of an empirical treatment that does not adhere to international guidelines is a risk factor independently associated with in-hospital mortality or development of adverse events. Several studies suggest improved outcomes among hospitalized CAP patients who are treated according to the CAP guidelines as opposed to those treated with a guideline-discordant regimen [12]. However, a possible association between adherence to the 2005 or 2007 ATS and IDSA guidelines for empirical antibiotic treatment and poor outcome is controversial; particularly, for patients with HCAP [12]. In fact, there is evidence showing an association between mortality and inappropriate empirical antibiotic treatment, defined as not receiving antibiotics with invitro activity against offending pathogens [10]. Furthermore, several reports show that HCAP patients infected with PDR pathogens are at high risk for inappropriate empirical antibiotic treatment $[9,10,13]$. This inappropriate empirical antibiotic treatment may be close to the empirical treatment that does not adhere to the 2005 or 2007 ATS and IDSA guidelines. However, Kett et al. [6, 14] recently report that compliance with the 2005 ATS/IDSA guidelines is associated with increased mortality, which has raised important questions for current recommendations for the management of nosocomial pneumonia in patients at risk for multidrug-resistant (MDR) pathogens that call for prompt broad-spectrum empirical treatment. In addition, a validation study by Nseir et al. [15] reports that the 2005 ATS/IDSA criteria for predicting infection or colonization related to MDR bacteria have a low positive predictive value, but an excellent negative predictive value, at ICU admission. Therefore, the recommendations in the 2005 ATS/IDSA guidelines remain controversial.

One group of researchers has disagreed with the concept of HCAP because it may mislead physicians into the administration of unnecessary broad-spectrum antibiotics [11]. In addition, it has been considered that HCAP should be broken down into several distinct subgroups in order to be able to use narrow-spectrum antibiotics [16]. However, physicians should at least be aware of those patients who are more likely to receive inappropriate empirical antibiotic treatment than patients with CAP because of the possible PDR pathogens, and should be able to distinguish these patients, like those with HCAP, from those with CAP $[9,10,12,13,17]$.

We should pay attention to the fact that PDR or MDR pathogens may not be the infectious agents in all patients with HCAP, if we choose to identify patients with HCAP [18]. That is, for patients with HCAP, there are both patients those who require broad-spectrum antibiotics and those who do not. Even if the definition of HCAP is further elucidated, this issue will probably persist. Furthermore, we must consider that the incidence of PDR pathogens occurrence is different in different countries and institutions [19]. There have been few prospective studies that have addressed the question, "Who should receive broad-spectrum antibiotics, and who does not need them among patients with HCAP?" Therefore, additional research is needed.

One important aim is to be able to appropriately manage patients with pneumonia, including antibiotic selection and systemic management (e.g., for complications, nutritional care, and prevention of aspiration). To achieve this aim, how should we assess or classify patients with pneumonia? This study has raised important concerns related to the assessment of severity for patients with pneumonia and the appropriate treatment strategies. The management of patients with pneumonia has changed over time. We are now facing a new era for reconsidering the management of patients with pneumonia.

Conflict of interest None.

\section{References}

1. Falcone M, Corrao S, Venditti M et al (2011) Performance of PSI, CURB-65, and SCAP scores in predicting the outcome of patients with community-acquired and healthcare-associated pneumonia. Intern Emerg Med. doi:10.1007/s11739-011-0521-y 
2. Fine MJ, Auble TE, Yealy DM et al (1997) A prediction rule to identify low-risk patients with community-acquired pneumonia. N Engl J Med 336:243-250

3. Lim WS, van der Eerden MM, Laing R et al (2003) Defining community acquired pneumonia severity on presentation to hospital: an international derivation and validation study. Thorax 58:377-382

4. Espana PP, Capelastegui A, Gorordo I et al (2006) Development and validation of a clinical prediction rule for severe communityacquired pneumonia. Am J Respir Crit Care Med 174:1249-1256

5. Venditti M, Falcone M, Corrao S et al (2009) Outcomes of patients hospitalized with community-acquired, health careassociated, and hospital-acquired pneumonia. Ann Intern Med 150:19-26

6. American Thoracic Society, Infectious Diseases Society of America (2005) Guidelines for the management of adults with hospital-acquired, ventilator-associated, and healthcare-associated pneumonia. Am J Respir Crit Care Med 171:388-416

7. Mandell LA, Wunderink RG, Anzueto A et al (2007) Infectious Diseases Society of America/American Thoracic Society consensus guidelines on the management of community-acquired pneumonia in adults. Clin Infect Dis 44(Suppl 2):S27-S72

8. Shindo Y, Sato S, Maruyama E et al (2008) Comparison of severity scoring systems A-DROP and CURB-65 for communityacquired pneumonia. Respirology 13:731-735

9. Shindo Y, Sato S, Maruyama E et al (2009) Health-care-associated pneumonia among hospitalized patients in a Japanese community hospital. Chest 135:633-640

10. Micek ST, Kollef KE, Reichley RM et al (2007) Health careassociated pneumonia and community-acquired pneumonia: a single-center experience. Antimicrob Agents Chemother 51:35683573
11. Ewig S, Welte T, Chastre J et al (2010) Rethinking the concepts of community-acquired and health-care-associated pneumonia. Lancet Infect Dis 10:279-287

12. Zilberberg MD, Shorr AF (2011) Healthcare-associated pneumonia: the state of evidence to date. Curr Opin Pulm Med 17:142-147

13. Park HK, Song JU, Um SW et al (2010) Clinical characteristics of health care-associated pneumonia in a Korean teaching hospital. Respir Med 104:1729-1735

14. Kett DH, Cano E, Quartin AA et al (2011) Implementation of guidelines for management of possible multidrug-resistant pneumonia in intensive care: an observational, multicentre cohort study. Lancet Infect Dis 11:181-189

15. Nseir S, Grailles G, Soury-Lavergne A et al (2009) Accuracy of American Thoracic Society/Infectious Diseases Society of America criteria in predicting infection or colonization with multidrug-resistant bacteria at intensive-care unit admission. Clin Microbiol Infect 16:902-908

16. Yu VL (2011) Guidelines for hospital-acquired pneumonia and health-care-associated pneumonia: a vulnerability, a pitfall, and a fatal flaw. Lancet Infect Dis 11:248-252

17. Kollef MH, Shorr A, Tabak YP et al (2005) Epidemiology and outcomes of health-care-associated pneumonia: results from a large US database of culture-positive pneumonia. Chest 128:3854-3862

18. Brito V, Niederman MS (2009) Healthcare-associated pneumonia is a heterogeneous disease, and all patients do not need the same broad-spectrum antibiotic therapy as complex nosocomial pneumonia. Curr Opin Infect Dis 22:316-325

19. Shindo Y, Hasegawa Y (2011) Healthcare-associated pneumonia among hospitalized patients. In: Esquinas A (ed) Applied technologies in pulmonary medicine. Karger Medical and Scientific Publishers, Basel, pp 172-177 